MonTENEGRo LeZA, S., «El antes y el después de la Ley Orgánica 3/2007», REDUR I4, diciembre 20i6, págs. II7-I36. ISSN i695-078X

\title{
EL ANTES Y EL DESPUÉS DE LA LEY ORGÁNICA 3/2007
}

\author{
Sofía MONTENEGRO LEZA
}

INVESTIGADORA PRE-DOCTORAL EN TRABAJO SOCIAL

UNIVERSIDAD DE LA RIOJA

SuMARIo: I. Introducción II. Precedentes legislativos III. Exposición de motivos para la redacción de la Ley Orgánica 3/2007 IV. Áreas reguladas en el texto legal V. Datos y clasificaciones del 2006-2016 VI. Conclusiones ¿Hemos llegado a nuestros objetivos? VII. Bibliografía

RESUMEN: El presente artículo examina el término igualdad de género contenido y desarrollado en la Ley Orgánica $3 / 2007$, de 22 de marzo, para la igualdad efectiva de mujeres y hombres. Igualmente este trabajo elabora una comparativa entre lo regulado en la norma y los avances sociales acaecidos en el periodo 2006-2016.

Palabras Clave: Igualdad, género, normativa, sociedad

ABSTRACT: The present article examines the Equality of Gender contained and developed in the Organic Law 3/2007, of March 22nd, for the effective equality of women and men. Besides this work elaborates a comparative among what has been regulated in the norm and the social advances happened in the period 2006-2016.

KEYwORDS: Equality, Gender, Normative, Society

\section{Introducción}

En nuestro ordenamiento jurídico se han consolidando paulatinamente diferentes aspectos otorgando a la mujer una posición más igualitaria respecto al hombre. Los logros de las mujeres están patentes en las sociedades actuales, en las cuales las mujeres escalan peldaños en multitud de facetas. España no es una excepción de este avance cuyo punto álgido tuvo lugar con la aprobación de la Ley Orgánica 3/2007, de 22 de marzo, para la igualdad efectiva de mujeres y hombres. ${ }^{\mathrm{I}}$. Dicho hecho evidenció públicamente la brecha existente entre hombres y mujeres y el afán del Estado por modificar esa situación.

\footnotetext{
${ }^{\mathrm{I}}$ Ley Orgánica 3/2007, de 22 de marzo, para la igualdad efectiva de mujeres y hombres (BOE num.7I, de 23 de marzo de 2007).
} 
Por primera vez en la historia legislativa de nuestro país, se realizó una toma de conciencia parlamentaria entendiendo el género como un elemento transversal de nuestra sociedad y nuestra normativa. Convirtiéndose, a su vez, en el texto legal de referencia a la hora de analizar cualquier situación relativa al género.

Diez años después de su aprobación, se requiere un ejercicio de evaluación para saber si se han conseguido los objetivos marcados. Para ello, se va a realizar una comparativa a partir de diferentes fuentes documentales para elaborar un marco al partir del cual poder analizar lo sucedido en estos diez años.

\section{Precedentes legislativos}

La Ley Orgánica 3/2007 fue el fruto del desarrollo del principio jurídico universal de igualdad entre mujeres y hombres. Pero es necesario señalar que, con carácter previo, España había vivido avances en materia de igualdad. La Ley Orgánica 3/2007 se convierte en la consecuencia histórica y social del esfuerzo de las generaciones predecesoras. Un ejemplo claro de ello fue la aprobación de la Constitución de I978 que supuso un antes y un después en muchos aspectos, también en lo relativo al género. La mujer dejaba de estar supeditada a los varones de su familia. De este modo se convertía en sujeto individual con derechos individuales.

Este valor es avalado tanto a nivel internacional como nacional. En nuestro caso, la primera referencia que podemos encontrar está en el artículo i4 de la Constitución Española «Los españoles son iguales ante la ley, sin que pueda prevalecer discriminación alguna por razón de nacimiento, raza, sexo, religión, opinión o cualquier otra condición o circunstancia personal o social». Un año después, en 1979, fue la Asamblea General de Naciones Unidas quien desarrolló este principio jurídico. A tal motivo, elaboró el texto denominado: Convención sobre la eliminación de todas las formas de discriminación contra la mujer. Cuatro años después España ratificó su contenido asumiéndola como parte de su ordenamiento. Dentro de la Unión Europea hubo que esperar hasta I999 año en el cual entró en vigor el Tratado de Ámsterdam. En dicho tratado se marcaba como objetivo de la Unión la integración del valor de la igualdad entre géneros en todas las políticas y acciones tanto propias como de sus miembros. Dicha intencionalidad se vio reforzada un año más tarde con la aprobación de la Carta de Derechos Fundamentales de la Unión, texto en el cual se otorgaba una nueva y renovada importancia al concepto de igualdad. Pero previamente a todos estos actos legales de reconocimiento del valor jurídico y superior que supone la igualdad, en I948 ya se orientaba hacia los objetivos que a día de hoy nos marcamos. Su marco no fue otro que La Declaración Universal de Derechos Humanos. En dicho texto emblemático internacional se reconoce en su artículo I que «Todos los seres humanos nacen libres e iguales en dignidad y derechos y, dotados como están de razón y conciencia, deben comportarse fraternalmente los unos con los otros».

A modo recopilatorio, se va a dejar enunciado una serie de doctrina jurídica respeto a la igualdad tanto a nivel nacional, europeo como internacional. 
- Textos Internacionales

- Carta de las Naciones Unidas.

- Declaración Universal de los Derechos Humanos.

- Pacto Internacional de Derechos Civiles y Políticos.

- Pacto Internacional de Derechos Económicos, Sociales y Culturales.

- Convención sobre la eliminación de todas las formas de discriminación contra la mujer.

- Protocolo Facultativo de la Convención sobre la eliminación de todas las formas de discriminación contra la mujer.

- Convenio $\mathrm{n}^{\circ}$ III de la Organización Internacional del Trabajo, relativo a la discriminación en materia de empleo y ocupación.

- Normativa Europea

- Convenio Europeo para la protección de los Derechos Humanos y Libertades Fundamentales.

○ Carta Social Europea.

- Protocolo adicional a la Carta Social Europea.

- Carta de los Derechos Fundamentales de la Unión Europea.

- Directiva 2000/78/CE del Consejo, de 27 de noviembre de 2000, relativa al establecimiento de un marco general para la igualdad de trato en el empleo y la ocupación.

- Directiva 2004/II3/CE del Consejo, de I3 de diciembre de 2004, por la que se aplica el principio de igualdad de trato entre hombres y mujeres al acceso de bienes y servicios y su suministro.

- Directiva 2006/54/CE del Parlamento Europeo y del Consejo, de 5 de julio de 2006, relativa a la aplicación del principio de igualdad de oportunidades e igualdad de trato entre hombres y mujeres en asuntos de empleo y ocupación.

- Reglamento (CE) n ${ }^{\circ}$ I922/2006 del Parlamento Europeo y del Consejo, 20 de diciembre de 2006 , por el que se crea un Instituto Europeo de Igualdad de Género.

- Normativa Nacional

- Ley 39/1999, de 5 de noviembre, para promover la conciliación de la vida familiar y laboral de las personas trabajadoras (BOE núm. 266, de 6 de noviembre de 1999)

- Ley 27/2003, de 3I de julio, reguladora de la orden de protección de las víctimas de violencia doméstica (BOE núm. I83, de I de agosto de 2003) 
- Ley 30/2003, de I3 de octubre, sobre medidas para incorporar la valoración de impacto de género en las disposiciones normativas que elabore el Gobierno (BOE núm. 246, de I4 de octubre de 2003)

- Ley Orgánica I/2004, de 28 de diciembre, de medidas de protección integral contra la violencia de género (BOE núm.3I3, de 29 de diciembre de 2004)

- Por lo tanto, el elemento innovador y diferenciador de la Ley Orgánica 3/2007 respecto a la legislación precedente fue la creación de un marco normativo en el que el tratamiento de la Igualdad de Género fue integral. Con importancia suficiente para tener una legislación propia a partir de la cual se regulara los elementos de otros ámbitos a partir del concepto de Igualdad de Género.

\section{Razones para la redacción de la Ley Orgánica 3/2007}

El nacimiento de esta ley fue la evidencia, reconocida por el Estado, de que los objetivos marcados en la Constitución y en los diferentes textos legislativos no habían sido suficiente para cumplir el principio de igualdad entre hombres y mujeres. Tomando como punto de partida la Exposición de Motivos de la Ley Orgánica 3/2007, algunas de las razones que empujaron a la aprobación de este marco legislativo fueron algunos como la violencia de género, la brecha salarial, la discriminación en lo relativo a las pensiones de viudedad, entre otras. Para hacernos una idea de a qué realidad estaban haciendo referencia, vamos a presentar unos datos recogidos en diferentes fuentes con el fin de contextualizar de la situación de la mujer en el 2006 respecto a la del hombre. A tal fin, hemos recurrido a diversas fuentes de información para vislumbrar algunos de los parámetros en los que hombres y mujeres ocupaban posiciones diferentes.

Tabla I. Contextualización de la situación de la mujer en el año 2006.

\begin{tabular}{|c|c|c|c|}
\hline ÁREA & MUJERES & HOMBRES & Fuente \\
\hline Tasa de paro & II, $6 \%$ & $6,3 \%$ & \multirow{5}{*}{$\begin{array}{l}\text { Anuario de } \\
\text { Estadísticas } \\
\text { Laborales y de } \\
\text { Asuntos Sociales } \\
2006 \\
\text { Diario el Mundo } \\
(27-0 \text { - 2007) }\end{array}$} \\
\hline Tasa de actividad & $48 \%$ & $69,3 \%$ & \\
\hline Trabajadores/as contratos temporales & $\mathrm{II}, 3 \%$ & $7,7 \%$ & \\
\hline $\begin{array}{l}\text { Pensiones contributivas (importe } \\
\text { medio) }\end{array}$ & 492,40 Euros & 798,05 Euros & \\
\hline $\begin{array}{l}\text { Número de prestaciones por } \\
\text { maternidad }{ }^{3} \text { según sexo del preceptor }\end{array}$ & $3 \mathrm{I} 7 \cdot 3 \mathrm{I} 8$ & 5.282 & \\
\hline
\end{tabular}

\footnotetext{
${ }^{2}$ Diario elmundo.es La tasa de desempleo cierra 2006 en el 8,3\%, el nivel más bajo desde 1979 (27/0I/2007) Disponible en: http://www.elmundo.es/mundodinero/2007/0I/26/economia/II69798824.html; fecha de consulta 2I.XII.20I6

${ }^{3}$ En el año 2006 la baja de maternidad era propia de la mujer aunque ella pudiera decidir, conforme al progenitor varón, compartir el periodo de baja entre ambos progenitores. Es por ello que en el informe se recoge bajo el concepto «baja de maternidad» la disfrutada por las madres y los padres. También es necesario indicar que la mujer está obligada, en caso de encontrarse empleada o desarrollando cualquier actividad laboral, a permanecer cuatro semanas de baja por motivos médicos. Por lo que cualquier mujer que trabaje tendrá la obligación de acogerse a esta baja.
} 


\begin{tabular}{|c|c|c|c|c|}
\hline \multicolumn{2}{|l|}{$\begin{array}{l}\text { Mujeres asesinadas: } \\
\text { Violencia de género }\end{array}$} & 70 & - & El País (28-I2-06) ${ }^{4}$ \\
\hline \multicolumn{2}{|c|}{ Tasa riesgo de pobreza (2004) } & $20,8 \%$ & $19,9 \%$ & \multirow{4}{*}{$\begin{array}{l}\text { Anuario estadístico } \\
2006 \text { INE }\end{array}$} \\
\hline \multirow{3}{*}{$\begin{array}{l}\text { Empleo de tiempo } \\
\text { a determinadas } \\
\text { actividades: }\end{array}$} & Trabajo & $25,2 \%$ & $43,3 \%$ & \\
\hline & $\begin{array}{l}\text { Hogar y } \\
\text { familia }\end{array}$ & $92,7 \%$ & $70 \%$ & \\
\hline & $\begin{array}{l}\text { Trabajo } \\
\text { voluntario y } \\
\text { reuniones }\end{array}$ & $\mathrm{I} 5, \mathrm{I} \%$ & $9,5 \%$ & \\
\hline \multicolumn{2}{|c|}{ Salario bruto medio anual } & I6.245,I7 Euros & 22.05I,O8 Euros & $\begin{array}{l}\text { Encuesta de } \\
\text { Estructura salarial } \\
\text { 2006. Resultados } \\
\text { definitivos }\end{array}$ \\
\hline
\end{tabular}

Fuente: Elaboración propia.

En este contexto se evidencia las circunstancias tan diferenciadas en las que hombres y mujeres se veían inmersos. En primer lugar y en lo relativo al ámbito laboral, las mujeres tenían un 5,3\% más de tasa de paro que sus compañeros varones, teniendo en cuenta que su participación en el mundo laboral era un 2I,3\% menor que la de los hombres. Además las mujeres trabajaban un 3,6\% más en contratos temporales que su compañeros. En síntesis, desde el punto de vista laboral las mujeres participaban menos. Dentro de la población activa eran ellas quienes vivían más situaciones de paro. Y, además, eran las mujeres quienes ostentaban más contratos temporales respecto a sus varones. Con todo ello, parece lógico pensar que la diferencia salarial media anual es derivada de todas estas circunstancias. Por lo que la brecha salarial no estará tanto fundada por la diferencia salarial desarrollando el mismo puesto, sino por la variedad de circunstancias que afectan a mujeres y hombres y que se traduce, finalmente, en diferencias salariales en cómputos globales.

Si pasamos a analizar la corresponsabilidad, entendiéndola como la responsabilidad compartida entre los componentes de una misma pareja en relación a las tareas del hogar y el cuidado de las terceras personas, puede verse como las mujeres son las protagonistas principales de las tareas relacionadas con el cuidado y el hogar. Eso puede verse tanto en el tiempo destinado a las tareas del hogar y la familia.

En este contexto, se tomó la decisión de llevar a cabo la realización de una acción normativa que combatiera cualquier tipo de manifestación de discriminación, de manera directa o indirecta, por razón de género. Ya que los datos evidenciaban que la igualdad era

\footnotetext{
${ }^{4}$ Diario El País. 70 mujeres muertas en 2006 (28/12/2006). Disponible en: http://sociedad.elpais.com/sociedad/2006/I2/28/actualidad/ıI67260406_850215.html; fecha de consulta 2I.XII.20I6.
} 
un principio jurídico asumido pero sin la transformación social al nivel deseado y establecido.

Otro elemento innovador consecuencia de la Ley Orgánica 3/2007 fue la prevención como materia legal en igualdad de género y la serie de «políticas activas» que pudieran hacer efectivo el principio de igualdad. De este modo, se potenció el valor transversal y transdisciplinar que toma forma a partir de este texto legislativo. Por lo tanto, estamos ante una normativa que no sólo eleva la importancia del concepto de igualdad si no que lo extrapola a todos los ámbitos dependientes de los organismos públicos y/o entidades privadas.

Un elemento, enmarcado dentro de las «políticas activas» que puede encontrarse a lo largo de toda la ley, es el denominado como «acciones positivas». Dicho concepto viene a significar un tratamiento especial de las mujeres en determinadas situaciones contempladas en el marco legal. Tradicionalmente este tipo de acciones eran denominadas «discriminaciones positivas» las cuales son definidas por la Real Academia Española como «Protección de carácter extraordinario que se da un grupo social históricamente discriminado». Dicho concepto tiene su origen en el Derecho antidiscriminatorio el cual surgió como reacción a las protestas protagonizadas por la población afroamericana, así como por parte de otras minorías en la década de los años 60 en Estados Unidos. En general, entendemos por acción o discriminación positiva aquel conjunto de medidas o planes vinculados al derecho cuyo objetivo final es la eliminación de la discriminación intergrupal ${ }^{6}$. Es decir, el concepto de discriminación está directamente vinculado al término de igualdad. Sin uno no se entiende el otro. Por lo tanto, es necesario tener en cuenta una serie de consideraciones para entender la realidad en la que se encuentra ambos conceptos ${ }^{7}$ . Por un lado, son conceptos relacionales que no pueden ser entendidos fuera de un contexto social en el que existe interacción entre sus componentes. Por otro lado, la igualdad no rechaza la existencia de las diferencias entre sujetos. Es decir, se acepta la necesidad de tratar de manera diferente a quienes son diferentes y la diferenciación se basa en los conceptos de necesidad, idoneidad y proporcionalidad. Por lo que rechaza las diferenciaciones arbitrarias que suponen diferencias de trato.

Desde el punto de vista de los Derechos Humanos se entiende que este concepto implica la atribución de una serie de prerrogativas a las personas que, por diversas circunstancias, se encuentren en una situación y/o posición desfavorecida dentro de la sociedad. Para ello, es defendido que para garantizar la igualdad entre sujetos es necesario, en ocasiones, establecer una serie de medidas por medio del trato diferenciado más favorable para los sectores que sean discriminados socialmente. En definitiva, los

\footnotetext{
${ }^{5}$ Diccionario de la Real Academia Española, www.rae.es (20I6)

${ }^{6}$ BARRÈre UnZueTA. M.A., «Igualdad y Discriminación Positiva: Un esbozo de análisis teórico-conceptual», Cuadernos electrónicos de filosofía del derecho, 9, 2003, Valencia. (disponible en

http://www.uv.es/cefd/9/barrereI.pdf; fecha de consulta:2I.XII.20i6).

${ }^{7}$ Bustos BotTAI, R., «Discriminación por razón de sexo y acciones positivas: reflexiones a la luz de la jurisprudencia constitucional española y aproximación a la ley para la Igualdad Efectiva», Revista de Filosofía, Derecho y Política, 6, 2007, págs.I27-I47 (disponible en http://universitas.idhbc.es/no6/o6-o8.pdf; fecha de consulta: 2I.XII.20I6).
} 
argumentos pueden agruparse $\mathrm{en}^{8} \mathrm{su}$ carácter deontológico por medio de las medidas compensatorias de las injusticias y desigualdades. Y, por otro lado, por su carácter teleológico por medio de las medidas que buscan la integración social, ya sea por utilidad social o por ideales solidarios.

Independiente de su carácter, este tipo de medidas no han estado exentas de polémicas. Ello se debe a que por su naturaleza supone el trato mejorado hacia un colectivo frente al perjuicio del otro sector. Por ello, se ha procurado tener especial cuidado exigiendo que sean excepcionales y que cumplan criterios de flexibilidad y objetividad. Además, se entiende que deben ser disposiciones transitorias hasta que sea alcanzado el objetivo marcado. Por lo tanto, cualquier medida de discriminación positiva tiene que ser entendida con carácter temporal y no indefinido. ${ }^{9}$

En definitiva, la Ley Orgánica 3/2007 es el resultado de una serie de medidas de diferente naturaleza que persigue el objetivo de que en España la igualdad entre géneros sea una realidad. Para ello, dentro del texto legal se trabaja en diferentes áreas sociales tales como la laboral, educativa, representación política entre otras. Con el fin último de ser una respuesta integral y transversal ante una situación problema que afecta a todos los estamentos de la sociedad.

\section{IV. Áreas reguladas en el texto legal}

La Ley Orgánica 3/2007 tiene como objetivo ser un elemento generalista que sea capaz de dar respuesta y cabida a cualquier situación problema que se genere en relación al género. De este modo, se conceptualiza como punto de partida general y a su vez especifica multitud de situaciones en las que se requieren acciones positivas para lograr la igualdad de género.

Por lo tanto, la Ley hace referencia a una gran gama de políticas públicas. Las cuales son desarrolladas a partir de los mandatos dados desde la Constitución a partir de la cual se otorga al Estado de las competencias para regular las condiciones básicas que garanticen la igualdad (Art. 9.2 C.E).

Con el objetivo de ofrecer una visión panorámica del texto legal, a continuación se presenta una tabla en la que se recoge sus componentes principales. De este modo, puede verse la multitud de áreas que son analizadas y articuladas.

\footnotetext{
${ }^{9}$ Giménez Mercado, C. Valente Adarme, X «El enfoque de los derechos humanos en las políticas públicas: ideas para un debate en ciernes», Cuadernos del Cendes, año 27, nº 74, Tercera Época, mayo-agosto 20 Io.
} 
Tabla 2. Esquema temático de la Ley Orgánica 3/2007, de 22 de marzo, para la igualdad efectiva de mujeres y hombres

\begin{tabular}{|c|c|}
\hline TITULOS & Áreas de Interés \\
\hline $\begin{array}{l}\text { I Principio de Igualdad y } \\
\text { la tutela contra la } \\
\text { discriminación }\end{array}$ & $\begin{array}{l}\text { - Empleo, formación, promoción y condiciones trabajo. } \\
\text { - Acoso sexual y acoso por razón de sexo. } \\
\text { - Embarazo y maternidad. } \\
\text { - Acciones positivas. }\end{array}$ \\
\hline $\begin{array}{l}\text { II Políticas Públicas para } \\
\text { la Igualdad }\end{array}$ & $\begin{array}{l}\text { - Transversalidad del principio igualdad. } \\
\text { - Nombramientos realizados por los Poderes Públicos. } \\
\text { - Planes Estratégicos, Informes, etc. } \\
\text { - Áreas: Educación, Salud, Producción artística, Deportes, Desarrollo Rural y } \\
\text { Urbano, etc. }\end{array}$ \\
\hline $\begin{array}{l}\text { III Igualdad y Medios de } \\
\text { Comunicación }\end{array}$ & $\begin{array}{l}\text { - Medios de Comunicación públicos y privados. } \\
\text { - Publicidad. }\end{array}$ \\
\hline $\begin{array}{l}\text { IV El Derecho al } \\
\text { Trabajo en Igualdad de } \\
\text { Oportunidades }\end{array}$ & $\begin{array}{l}\text { - Programas de mejoras de empleabilidad de mujeres. } \\
\text { - Promoción de la igualdad en la negociación colectiva. } \\
\text { - Derechos de conciliación de la vida personal, familiar y laboral. } \\
\text { - Planes de Igualdad. }\end{array}$ \\
\hline $\begin{array}{l}\text { V El principio de } \\
\text { igualdad en el empleo } \\
\text { público }\end{array}$ & $\begin{array}{l}\text { - Presencia equilibrada en la Administración. } \\
\text { - Formación. } \\
\text { - Prestaciones. }\end{array}$ \\
\hline $\begin{array}{l}\text { VI Igualdad de trato en } \\
\text { el acceso a bienes y } \\
\text { servicios y su suministro }\end{array}$ & $\begin{array}{l}\text { - Igualdad de trato en el acceso a bienes y servicios. } \\
\text { - Protección en situación de embarazo. }\end{array}$ \\
\hline $\begin{array}{l}\text { VII Igualdad en la } \\
\text { responsabilidad social de } \\
\text { las empresas }\end{array}$ & -Participación de mujeres en los Consejos de Administración. \\
\hline $\begin{array}{l}\text { VIII Disposiciones } \\
\text { organizativas }\end{array}$ & $\begin{array}{l}\text { - Comisión Interministerial Igualdad. } \\
\text { - Unidades de Igualdad. } \\
\text { - Consejo de Participación de la Mujer. }\end{array}$ \\
\hline
\end{tabular}

Fuente: Elaboración propia.

Revisando el texto legal se presume que la ley puede ser entendida como un código de conducta al que atenerse en las situaciones derivadas de la discriminación de género. Por lo tanto, se ha convertido finalmente en un conjunto de herramientas que requieren de un desarrollo para su cumplimiento. Dicha idea es explicada a partir de la exposición de motivos de la Ley Orgánica 3/2007 en la que se lee: «La ordenación general de las políticas públicas, bajo la óptica del principio de igualdad y la perspectiva de género, se plasma en el establecimiento de criterios de actuación de todos los poderes públicos (...)». Es decir, se pretende abarcar una gran diversidad de ámbitos de la vida social, estableciendo criterios de actuación para los cuales se requiere de un desarrollo ulterior. Asumiendo los efectos 
secundarios del principio de igualdad en esta regulación y teniendo como consecuencia la modificación en más de veinte leyes vigentes en aquel momento.

Tabla 3. Listado de leyes modificadas a partir de la aprobación de la Ley Orgánica 3/2007 por orden cronológico

Ley 36/I977, de 23 de mayo, de Ordenación de los Cuerpos Especiales Penitenciarios y de Creación del Cuerpo de Ayudantes de Instituciones Penitenciarias.

Ley 50/I98I, de 30 de diciembre, por la que se aprueba el Estatuto Orgánico del Ministerio Fiscal.

Ley I6/i983, de 24 de octubre, de creación del Instituto de la Mujer.

Ley 26/1984, de i9 de julio, General para la Defensa de Consumidores y Usuarios.

Ley 30/1984, de 2 de agosto, de Medidas para la Reforma de la Función Pública.

Ley Orgánica 6/ı985, de I de julio, del Poder Judicial.

Ley I4/ı986, de 25 de abril, General de Sanidad.

Ley de Sociedades Anónimas, texto refundido aprobado por Real Decreto Legislativo $1564 / 1989$, de 22 de diciembre.

Ley General de la Seguridad Social, aprobado por Real Decreto Legislativo I/1994, de 20 de junio.

Ley 25/I994, de I2 de julio, por la que se incorpora al ordenamiento jurídico español la Directiva $89 / 552 /$ CEE.

Ley del Estatuto de los Trabajadores, aprobado por Real Decreto Legislativo I/ı995, de 24 de marzo.

Ley 3I/I995, de 8 de noviembre, de Prevención de Riesgos Laborales.

Ley 29/1998, de I3 de julio, reguladora de la Jurisdicción Contencioso-Administrativa.

Ley I7/I999, de I8 de mayo, de Régimen del Personal de las Fuerzas Armadas.

Ley 42/I999, de 25 de noviembre, de Régimen del Personal del Cuerpo de la Guardia Civil.

Ley I/2000, de 7 de enero, de Enjuiciamiento Civil.

Ley sobre Infracciones y Sanciones en el Orden Social, aprobado por Real Decreto Legislativo 5/2000, de 4 de agosto.

Ley I2/200I, de 9 de julio, de Medidas Urgentes de Reforma del Mercado de Trabajo.

Ley 55/2003, del Estatuto Marco del Personal Estatutario de los Servicios de salud.

Ley I6/2003, de 28 de mayo, de Cohesión y Calidad del Sistema Nacional de Salud.

Ley 56/2003, de 16 de diciembre, de Empleo.

Fuente: Elaboración propia a partir de la Ley Orgánica 3/2007.

En otras palabras, la Ley Orgánica 3/2007 supone una visión de conjunto de todo lo que implica o le afecta la igualdad de género. Además, y tal y como se ha esbozado anteriormente, supone una reconceptualización de lo que significa la igualdad de género. Lo cual tuvo como consecuencia la gran cantidad de modificaciones de la estructura legal estatal. Todo ello para adecuar lo ya establecido a la nueva visión. Es decir, todo ello para adaptar el marco legal a una nueva perspectiva, la perspectiva de género. La cual puede 
definirse $^{\text {ro }}$ como el reconocimiento de que la diferencia sexual es diferente a las atribuciones, ideas, representaciones y prescripciones sociales que se construyen a partir de esas diferencias sexuales. Por lo tanto la perspectiva de género es la toma de conciencia de esas construcciones sociales para, a partir del momento en el que se es consciente, establecer una serie de políticas y/o acciones que eviten esos condicionamientos sociales discriminatorios e irracionales.

Por lo tanto, y en consecuencia, se requieren de algunas herramientas para completar y desarrollar los preceptos enunciados en la Ley Orgánica 3/2007. De este modo, se ha intentado dar respuesta a las necesidades sociales existentes en el contexto de la aprobación de la ley. Algunas de esas herramientas son:

- Informes de Impacto de Género en los Presupuestos.

- Planes Estratégicos de Igualdad de Oportunidades.

- Informes por ministerios sobre la aplicación del Principio de Igualdad.

- Medidas concretas en cada ámbito, por ejemplo: Permiso por paternidad independiente del de maternidad; listas cremallera; cuotas establecidas; etc.

- Creación de un Ministerio de Igualdad (a día de hoy está dentro del: Ministerio de Sanidad, Servicios Sociales e Igualdad)..

- Separación de la Violencia de Género y la Igualdad de Oportunidades.

- Consejos para diferentes fines.

- Publicaciones periódicas.

- Creación Unidades de Igualdad.

- Campañas de sensibilización.

Por lo tanto, y tal y como hemos venido explicando, la Ley Orgánica 3/2007 aborda una gran variedad de postulados y premisas con el objetivo de cambiar la realidad entre los géneros. De la promulgación de este texto ha pasado casi una década. Entendiendo que toda ley busca el cambio social en un periodo de corto medio plazo, tras diez años se requiere un estudio de la situación en el momento actual.

\section{Datos y clasificaciones del 2006-2016}

A la hora de estudiar la situación actual vamos a fijar nuestro análisis en dos líneas muy concretas. La primera de ellas va a consistir en contextualizar la realidad de la mujer del mismo modo que se realizó anteriormente para el año 2006. La segunda va a basarse en el informe anual publicado por World Economic Forum - Committed to improving the state of the world. Dichos informes se denominan The Global Gender Gap Report.

\footnotetext{
${ }^{\text {ro }}$ LAMAS, M. «Diferencias de sexo, género y diferencia sexual», Cuicuilco, 7, enero-abril 2000.
} 
Previamente al análisis de la tabla que se presenta, es necesario indicar que algunos datos han sido sacados de la misma. Por lo tanto, no puede verse la comparativa entre los dos años. La razón ha sido que no se han encontrado los datos actualizados debido a que no se han publicado para el 2015 .

Tabla 4. Comparativa entre la contextualización de la situación de la mujer en 2006 y en 2015.

\begin{tabular}{|c|c|c|c|c|c|}
\hline \multirow{2}{*}{ ÁREA } & \multicolumn{2}{|c|}{ MUJERES } & \multicolumn{2}{|c|}{ HOMBRES } & \multirow[b]{2}{*}{ Fuente } \\
\hline & 2006 & 2015 & 2006 & 2015 & \\
\hline Tasa de paro & II, $6 \%$ & $23,55 \%$ & $6,3 \%$ & $20,77 \%$ & \multirow{4}{*}{$\begin{array}{l}\text { Anuario de } \\
\text { Estadísticas } \\
\text { Laborales y de } \\
\text { Asuntos } \\
\text { Sociales } 2006 \\
\text { y } 2015 . \\
\text { Anuario } \\
\text { estadístico } \\
\text { 20I6 INE }\end{array}$} \\
\hline Tasa de actividad & $48 \%$ & $53,7 \%$ & $69,3 \%$ & $65,69 \%$ & \\
\hline $\begin{array}{l}\text { Pensiones contributivas (importe } \\
\text { medio) }\end{array}$ & $\begin{array}{l}492,40 \\
\text { Euros }\end{array}$ & $\begin{array}{l}683,40 \\
\text { Euros }\end{array}$ & $\begin{array}{l}798,05 \\
\text { Euros }\end{array}$ & $\begin{array}{l}\text { I.100,67 } \\
\text { Euros }\end{array}$ & \\
\hline $\begin{array}{l}\text { Número de prestaciones por } \\
\text { maternidad/ paternidad según } \\
\text { sexo del preceptor }\end{array}$ & 317.318 & 276.482 & 5.282 & 4.929 & \\
\hline $\begin{array}{l}\text { Mujeres asesinadas: Violencia de } \\
\text { género }\end{array}$ & 70 & 57 & - & - & $\begin{array}{l}\text { El país } \\
\text { (Diciembre del } \\
2006 \text { y del } \\
2015)\end{array}$ \\
\hline Tasa riesgo de pobreza & $20,8 \%$ & $22, \mathrm{I} \%$ & $19,9 \%$ & $22,4 \%$ & $\begin{array}{l}\text { Anuario } \\
\text { estadístico } \\
2006 \\
\text { INE }\end{array}$ \\
\hline Salario bruto medio anual & $\begin{array}{c}\text { I6.245,I7 } \\
\text { Euros }\end{array}$ & $\begin{array}{c}\text { I9.744,82 } \\
\text { Euros }\end{array}$ & $\begin{array}{c}22.05 \mathrm{I}, 08 \\
\text { Euros }\end{array}$ & $\begin{array}{c}25 \cdot 727,24 \\
\text { Euros }\end{array}$ & $\begin{array}{l}\text { Encuesta de } \\
\text { Estructura } \\
\text { salarial } 2006 \text { y } \\
2014 \text {. } \\
\text { Resultados } \\
\text { definitivos }\end{array}$ \\
\hline
\end{tabular}

Fuente: Elaboración propia

La situación de la mujer y del hombre, teniendo en cuenta estos datos, han variado. El contexto de crisis ha afectado a ambos géneros. Ejemplo de ello es la tasa de paro que se ha visto incrementada en ambos casos. Los hombres han visto crecer su tasa un $14,47 \%$ en estos años, mientras que en el caso de la mujer ha crecido un II,95\%. Ello da como resultado que la brecha existente entre hombres y mujeres halla decrecido siendo en 2015 de un $2,78 \%$ mientras que en 2006 existía una distancia del 5,3\%. En el caso de la tasa de actividad, las mujeres han aumentado su participación en el mundo laboral en un 5,7\%. Mientras que los varones la han visto reducirse un 3,6r\%. Por lo que las mujeres han aumentado su participación en la búsqueda de trabajo lo cual no ha ido unido a la obtención de puesto de trabajo a tenor de los datos. 
Otro aspecto que puede valorarse es el referido a las pensiones contributivas. En ellas podemos ver como tanto los hombres como las mujeres han visto aumentadas las cuantías. Las mujeres las han visto aumentar en un $38,78 \%$, mientras que los hombres han visto un aumento del 25,38\%. En términos relativos puede verse como los varones no se han visto tan beneficiados como las mujeres en este periodo, pero si se tienen en cuenta los ingresos medios en cuestión de pensiones contributivas podemos ver como los hombres siguen disfrutando de una situación más beneficiosa. Prueba de ello es que las mujeres cobran un $46,42 \%$ menos que los hombres. Dicha diferencia se ha visto reducida en estos últimos años pasando del $62 \%$ de brecha al $46,42 \%$. Aún habiéndose visto reducida la distancia sigue dejando patente las diferencias que sigue habiendo en materia de salarios. Debido a que son los sueldos quienes marcan las diferencias entre unas pensiones contributivas y otras.

Respecto a las prestaciones por maternidad y paternidad tenemos que aclarar un aspecto importante que cambio a raíz de la Ley Orgánica 3/2007. En el año 2006 los hombres que querían disfrutar de una baja por paternidad tenían que hacer uso y disfrute de una fracción de la de la progenitora. En cambio, en el 20I5, los hombres ya pueden disfrutar de su baja por paternidad independiente totalmente de la de la mujer. Es decir, ahora cada progenitor tiene derecho a una baja por el motivo de haber sido padre o madre independiente de la de su pareja o de la del otro progenitor. Dicho derecho del progenitor es entendido por la ley como «un derecho individual y exclusivo del padre, que se reconoce tanto en los supuestos de paternidad biológica como en los de adopción y acogimiento». Posteriormente, este derecho se vio ampliado llegando a poder disfrutar el padre de la suspensión de su contrato de trabajo por un periodo de cuatro semanas (20 días) ${ }^{\mathrm{II}}$. Explicado esto, queda decir que otorgar el derecho a los progenitores masculinos no ha venido de la mano de un uso elevado del mismo. No es necesario analizar las cifras para observar la gran distancia que hay entre mujeres y hombres. Ello puede entenderse por diferentes motivos:

I. Las mujeres tienen obligación de acogerse a una baja mínima.

2. Los hombres no tienen obligatoriedad de acogerse a ella.

3. Las bajas tienen consecuencias económicas en los salarios en general.

4. Si los hombres, de media, cobran más que las mujeres si éstos se acogiesen al derecho de baja perdería, de media, más poder adquisitivo en comparación con el que perderían en caso de que las mujeres fueran quienes lo cogieran.

5. La sociedad no desarrolla un modelo corresponsable en la distribución de tareas y roles.

\footnotetext{
${ }^{\text {II }}$ En octubre de 20I6, ha sido aprobado por el Parlamento la iniciativa que insta al Gobierno a que legisle para que la baja de ambos progenitores sea de 16 semanas, intransferible y Iо०\% remunerado. Disponible en http://politica.elpais.com/politica/20I6/ı/I8/actualidad/I4767850II_903428.html. Por otro lado, el I de enero entrará en vigor la ampliación de la baja por paternidad de 2 semanas a 4 . Disponible en

http://economia.elpais.com/economia/20I6/I2/I6/actualidad/I48I893II2_I27597.html
} 
Estos son sólo unos ejemplos que demuestran que aún queda mucho por hacer. Pero aparte de este análisis habiendo tenido en cuenta una gama de datos, vamos a hacernos eco del análisis realizado por World Economic Forum - Committed to improving the state of the world. Este organismo realiza un informe anual que analiza, a partir de los datos disponibles de unos 200 países, la situación en cuestión de género. A partir de ahí, y teniendo en cuenta multitud de variables, realizan una clasificación. El informe al que estamos haciendo referencia es el denominado The Global Gender Gap Report.

Lo primero que es necesario decir es que este organismo realiza el análisis de cuatro macro variables. Teniendo cada una de ellas, a su vez, otros grupos de variables que dan como resultado una puntuación a partir de una serie de cálculos estadísticos. Dichas puntuaciones oscilan entre o y I, siendo la unidad la puntuación máxima posible. A partir de todo ello, World Economic Forum establece un orden entre los diversos países de los que posee datos. De este modo, realiza un ranking a nivel mundial respecto a la igualdad de género. Dicha clasificación general se hace por medio de una serie de cálculos de cada una de las macro-variables. De dichos grupos de variables también se hacen rankings propios. Por lo tanto, se va a presentar una comparativa en el intervalo temporal del 2006 al 2016 respecto a cinco clasificaciones. Cuatro de ellas siendo las correspondientes a las macrovariables, mientras que la quinta es la clasificación mundial general.

En segundo lugar, es imprescindible explicar cuáles son los componentes temáticos de cada uno de los grupos de variables.

1. Economic Participation and Opportunity: Salarios, niveles de participación, acceso a un empleo de alta cualificación.

2. Educational Attainment: Resultados de acceso a la educación básica hasta la superior.

3. Health and Survival: Esperanza de vida y la proporción de sexos. En este caso no se asume la paridad ya que se considera que existe una brecha de género en el caso de que las mujeres vivan un $6 \%$ menos que los hombres. Ello se realiza de este modo debido a que estadísticamente las mujeres tienen mayor esperanza de vida que los varones en términos generales. Por lo que sólo en el caso planteado se entendería que existen circunstancias contrarias al bienestar de las mujeres y por lo tanto brecha de género.

4. Political Empowerment: Resultados sobre representación en las estructuras de toma de decisiones.

Para realizar la comparativa contextualizada en el periodo que estamos analizando, hemos estudiado los informes realizados por esta institución en el periodo 2006-2016. De este modo, pretendemos dar un estudio por parte de una Institución de prestigio y valorada internacionalmente.

Para este propósito, facilitamos cinco gráficos con los cuales se muestra la evolución en cada uno de los grandes apartados que analiza The World Economic Forum. El primero ellos hace referencia a las diferentes posiciones y puntuaciones que ha obtenido 
España para cada uno de los años. Pero sin entrar en matices relativos a variables. Es decir, las puntuaciones medias una vez tenidas en cuenta las diferentes áreas de medición.

Gráfico I. Comparativa de las posiciones y puntuaciones de España en los informes The Global Gender Gap Report del periodo 2006 -20I6

\section{General Global Classification}

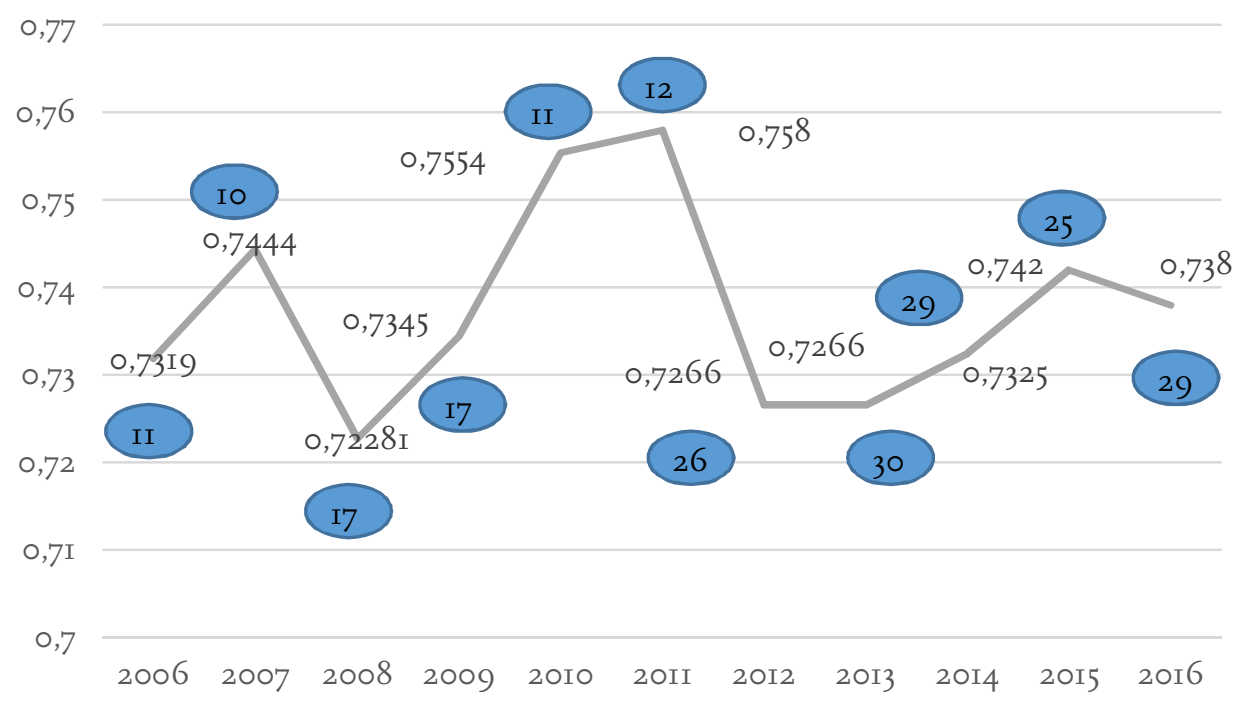

Fuente: Elaboración propia a partir de The Global Gender Gap Report

Como puede observarse, la variación de puestos que ha ostentando España oscila entre el ir y el 29. Sin que ello haya supuesto una diferencia amplia en términos absolutos. Ello se debe principalmente a que este informe anual no sólo tiene en cuenta los avances y los retrocesos de cada uno de los países, si no que los tiene en cuenta en comparación con otros países. Por ello, España ha estado en posiciones iguales teniendo puntuaciones diferentes. Ejemplo de ello son los años 2006 y 2010 en los cuales España se encuentra en la posición itcon dos puntuaciones que distan entre si 0,0235 . Por otro lado, el momento mejor de España en este informe fue en el año $2007(0,7444)$. En este año aún sin lograr sus mejores puntuaciones se encuentra en esta posición tanto por sus mejoras respecto al año anterior como por el empeoramiento o estancamiento de otros países.

Las principales causas que provocaron ese aumento, tanto en puntuación como en posición entre el año 2006 y el 2007, fueron debidos al área económica. Hubo dos subapartados que vieron aumentada su puntuación y que ocasionaron la subida en el escalafón. Dichas variables fueron: I. Ingresos: la diferencia entre hombres y mujeres, es decir la brecha salarial, se redujo de I7.468 a 16.897, es decir un 3,27\%; 2. Empleo de alta cualificación: el porcentaje de mujeres ocupando este tipo de puesto de trabajo aumentó un $2 \%$ respecto al año anterior. Estas dos casusas, aun teniendo en cuenta las variables en las que España obtuvo peor puntación, respecto al 2006, hicieron que escalara un puesto.

Como año a tener en cuenta, vamos a analizar el año 2008 debido a que no fuera el año con peor posición si es el periodo con menor puntuación. En este año se produjo una bajada generalizada en los cuatro grupos de variables. En el apartado económico descendió 
cinco puestos respecto al año anterior. El elemento más visible fue el relativo a los ingresos económicos. Ello debido a que volvió a crecer la brecha salarial. La diferencia respecto al 2007 fue de I.092\$. Dentro del ámbito educativo las puntuaciones se mantuvieron estables, por lo que debe entenderse que la bajada en posiciones se debió a la mejora en este apartado por parte de otros países. Lo mismo ocurre en el caso del ámbito de la salud, misma puntuaciones pero diferente posicionamiento. El apartado más destacado es el relativo a la representación política ${ }^{\mathrm{r2}}$. El principal motivo de cambio fue la celebración, ese año, de elecciones generales al Parlamento de España. Ello tuvo como efecto que bajara posiciones en las tres mediciones que tiene en cuenta, bajando a su vez en puntuaciones en dos de ellas. Las variables que mide y en las que desciende en puntuación son: I. Mujeres en el Parlamento y 2. Mujeres dentro del Consejo de Ministros/as. Dicho resultado evidencia una de las premisas de este artículo. La aprobación de textos legales en los que se contemplen avances sociales no se traduce en una sociedad más avanzada de manera automática. En el 2008, tras la aprobación de la Ley Orgánica 3/2007 nos encontramos en una situación más negativa que en el 2006.

Por otro lado, y aun no siendo el peor año en términos de puntuación, es necesario comentar que ocurrió en el año 20I3 para obtener la peor posición que, hasta el día de hoy. Durante este periodo hubo una subida generalizada aunque mínima en uno de los cuatro sub-grupos: Participación económica y oportunidad. Especialmente en los apartados de a) Participación de la fuerza laboral y b) Puestos de trabajo no altamente cualificados. Es decir, esto no significa que las mujeres se introdujeran en mayor medida en el ámbito laboral remunerado. Si no que, dentro del contexto de crisis, las fuerzas entre ambos géneros se acercaron al ser expulsados del marco laboral. A pesar de ese aumento de fuerzas en el ámbito laboral, la bajada en el ámbito de participación político es lo que se traduce en la posición ostentada ese año por España. Por lo tanto, la posición se entiende tanto por su puntuación como por la situación de mejoría de otros países respecto a años anteriores. Todo ello hizo que aun sin ser el peor año para España en términos totales, sí que lo fuera en términos relativos.

Para terminar este primer análisis, vamos a comparar la situación del 2006 con la del 20i6. Para poder exponer una visión y una comparación contextualizada en el tiempo. El análisis anual de cada variable se realizará a continuación. Los mayores cambios en estos diez años se traducen en: I.Subida en la puntuación (0,0०6) términos absolutos justificada por el aumento en las variable de participación económica. 2. Bajada o estancamiento del resto de áreas. Siendo notoria la bajada sufrida en el ámbito de representación política en la que hay una diferencia de o,I05. Ocasionándose la mayor bajada en el apartado de Mujeres en el Consejo de Ministros/as en el que se pasa de una segunda posición a la posición número 24, con una bajada en términos absolutos de 0,556.

\footnotetext{
${ }^{12}$ En el apartado correspondiente a la Participación Política de mujeres nos explayaremos en la explicación de este caso concreto.
} 
Tras este análisis general, pasamos a realizar un estudio por cada una de las subvariables. Para que, de manera gráfica, pueda visualizarse el recorrido que se ha tenido en cada uno de estos grupos.

El primero de ellos, referente a la Participación Económica y a las Oportunidades se contextualizada dentro de un marco de crisis. Sin embargo, este hecho no hace que la brecha entre hombres y mujeres se vea ampliada. Este acontecimiento, tal y como se ha anunciado anteriormente, se argumenta a partir del hecho de que se ha creado una igualdad en la base. Es decir, se han visto disminuidas las oportunidades tanto para hombres como para mujeres, por lo que se ha obtenido una situación más igual dentro de la negatividad de la misma. Pero a su vez, es notable el hecho de que ha visto mejorada las puntuaciones pero no las posiciones dentro de la clasificación. Ello se debe a que en otros países no sólo se reduce la brecha entre ambos géneros si no que se hace en pro de una participación mayor de la mujer.

Gráfico 2. Comparativa de las posiciones/puntuaciones de España en los informes The Global Gender Gap Report del periodo 2006 -2016 en el área «Economic Participation and Opportunity»

Economic Participation and Opportunity

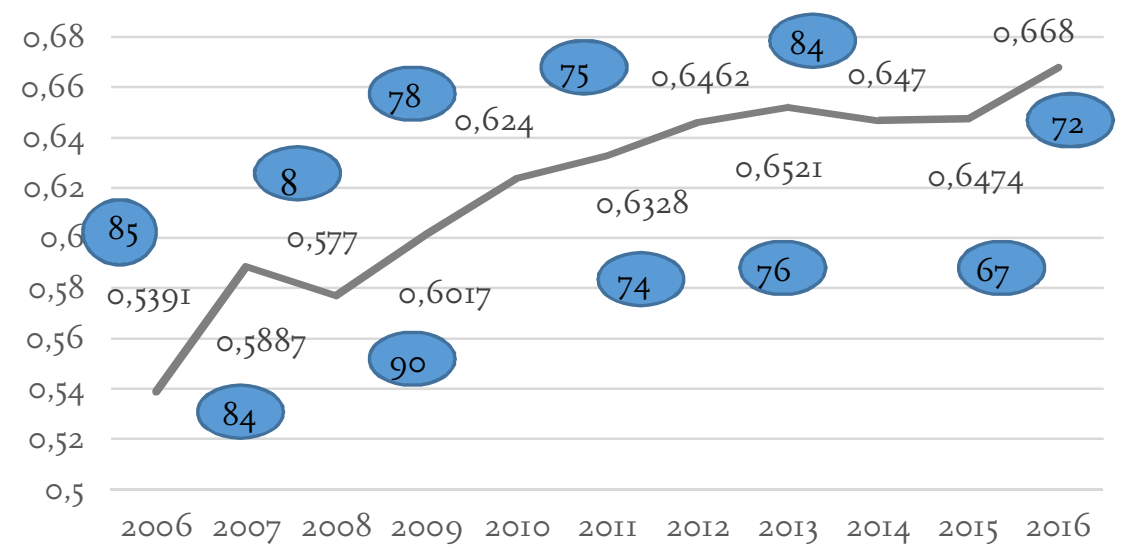

Fuente: Elaboración propia a partir de The Global Gender Gap Report.

Otro de los grandes apartados es el relativo a la Educación. En esta ocasión, España siempre está en puntuaciones muy elevadas teniendo en cuenta que éstas oscilan entre el o y el I. La razón por la que no ostentamos primeras posiciones se debe a que tiene que competir con países, principalmente del Norte de Europa, cuyos sistemas educativos acogen a una gran gama de ciudadanía, en muchos intervalos de edad, con un fracaso escolar menor que el nuestro. Ello hace que, aun teniendo una puntuación cercana al i no osténtennos posiciones más elevadas. 
Gráfico 3. Comparativa de las posiciones/ puntuaciones de España en los informes The Global Gender Gap Report del periodo 2006 -20I6 en el área «Educational Attainment»

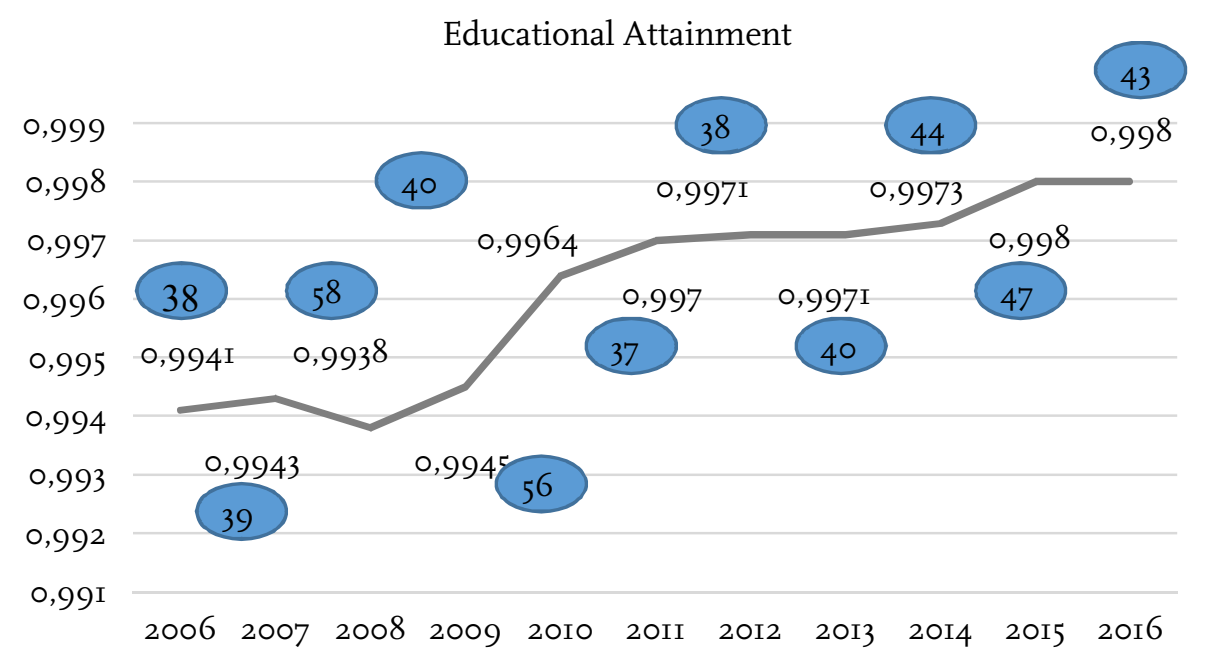

Fuente: Elaboración propia a partir de The Global Gender Gap Report

En lo relativo al ratio de niñas nacidas y a la esperanza de vida, sólo es pertinente decir que las puntuaciones oscilan entre 0,972 y el 0,979I por lo que se entiende que en cualquier caso es positivo desde la perspectiva de igualdad. Es en el apartado donde menores oscilaciones existen en lo relativo a las puntuaciones. Otra cosa es el tema de los posicionamientos los cuales nos hacen movernos desde la posición número 93 en el año 20I5, hasta la número 34 en el año 20I2. Ello se debe a la constante española que se ve afectada por el aumento o la disminución de puntuaciones de otros países.

Gráfico 4. Comparativa de las posiciones/ puntuaciones de España en los informes The Global Gender Gap Report del periodo 2006 -2016 en el área «Health and Survival»

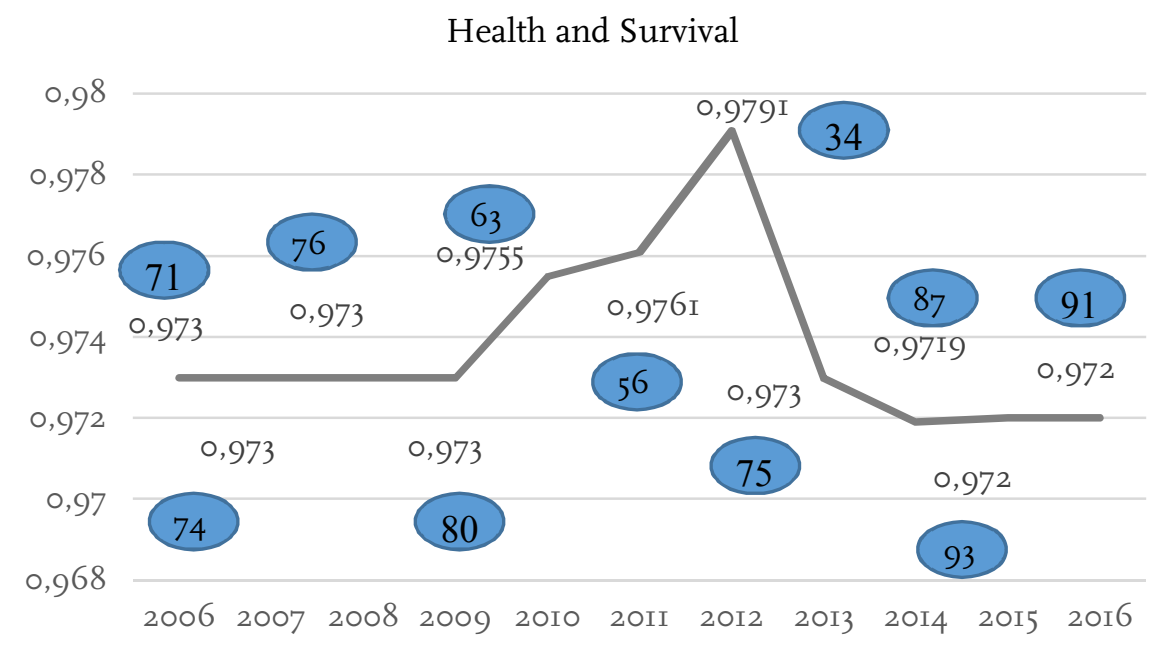

Fuente: Elaboración propia a partir de The Global Gender Gap Report 
El ámbito en el que existen mayores cambios, tanto en puntuaciones como en posiciones, es el de Empoderamiento Político. Por ello es en el apartado en el que mayor incidencia vamos a realizar. Ya que en el resto, aun habiendo cambios en las puntuaciones que es lo que hace referencia al hacer o al no hacer de España, se observa una tendencia afectada por los avances o retrocesos de otros países. Este contexto, no encaja con los datos que se desprenden de este último apartado.

Gráfico 5. Comparativa de las posiciones/puntuaciones de España en los informes The Global Gender Gap Report del periodo 2006 -2016 en el área «Political Empowerment»

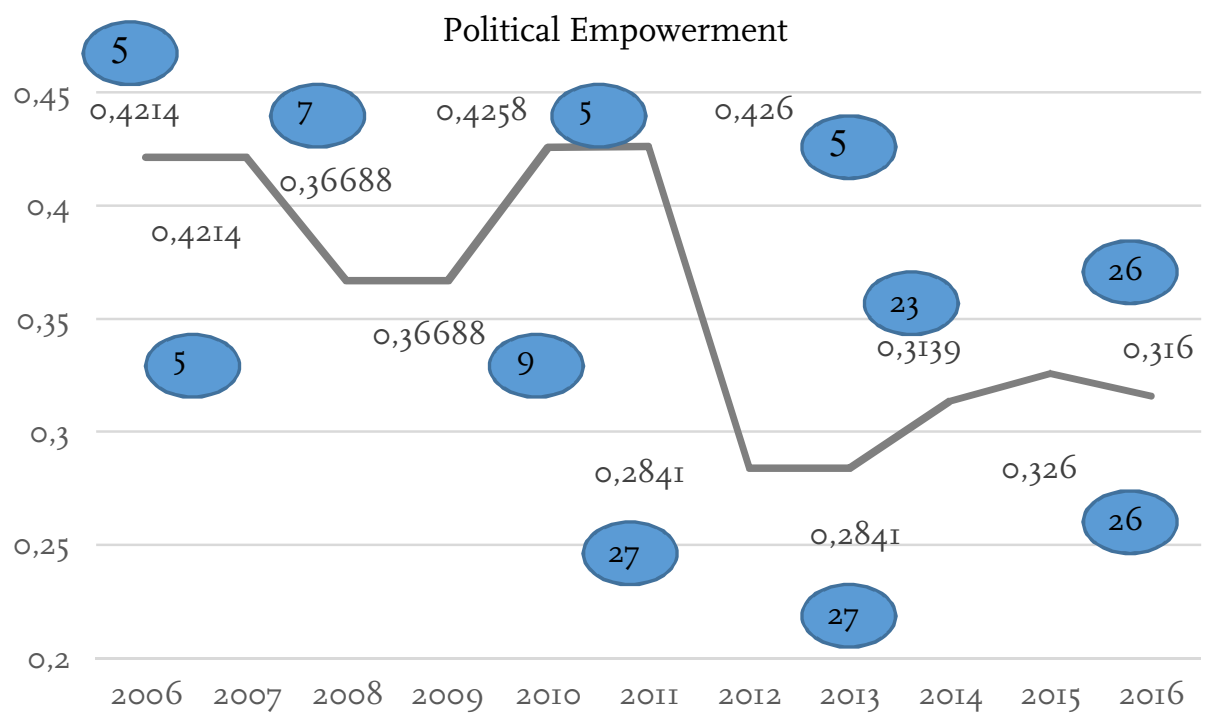

Fuente: Elaboración propia a partir de The Global Gender Gap Report.

Dentro de este marco temporal, partimos de una posición buena con una puntuación a medio camino entre la igualdad y la desigualdad. En el año 2006 obtuvimos buena puntuación, I,०० sobre I,००, en el apartado de Mujeres Ministras ya que eran el 50\% del total de ministros/as. Respecto a las mujeres dentro del Parlamento obtuvimos una puntuación 0,56 resultado de que las mujeres suponían un 36\% de la Cámara Legislativa. En lo referente a mujeres como cabezas del Estado, nunca ha ostentado ese puesto una mujer en los últimos 50 años por lo que las puntuaciones siempre eran ०,००. Por lo tanto, dicha variable no va a ser evaluada y no comentada en adelante. Con ello logramos una quinta posición la cual puede evaluarse como positiva entendiéndola como punto de partida y previa a la Ley Orgánica 3/2007. En el 2007 obtuvimos la misma puntuación y posición. Si hay que decir aumentamos una posición en lo relavo a número de Ministras pero por cambios externos a nosotros. El año 2008 fue negativo en lo relativo a la participación política femenina. Ese año hubo elecciones y el mapa político, en lo referente a mujeres representantes y ministras, cambió. La mayor bajada se evidenció en el número de Ministras (un 44\% frente a un 66\% de hombres en las cabezas de los Ministerios). Ese descenso hizo que bajáramos de tener I, ०० sobre I,०० a ०,78 sobre I,००. Dicha tendencia se mantuvo al año siguiente. Dicha circunstancia cabe ser analizada debido a que el Gobierno 
resultante de las elecciones fue el mismo que promulgo y desarrolló la Ley Orgánica $3 / 2007$.

En el 20Io, en cambio, la puntuación aumentó un 0,0589 debido a que volvió a haber un cambio en la composición del Consejo de Ministros/as traduciéndose en un aumento de mujeres al cargo de Ministerios. Teniendo como resultado un Ejecutivo con un $53 \%$ de Ministras frente a un $47 \%$ de Ministros. Todo ello se mantuvo hasta llegar al 2012. Las elecciones trajeron un cambio en el color del Gobierno, lo cual se tradujo en una bajada de mujeres a cargo de Ministerios. Pasamos de un 53\% en años anteriores a un 31\%, por lo que se evidenció notablemente el retroceso en este aspecto. A partir de este momento, la tendencia se ha mantenido aunque con cambios porcentuales. En el momento actual, ostentamos la posición número 26 con una puntuación del o,3i6. Siendo las mujeres un 40\% del Parlamento y un 31\% del Ejecutivo.

Este hecho evidencia que la Igualdad de Género requiere más que la aprobación de una serie de textos legales. En este caso, es el Gobierno y las Cortes quienes tienen que garantizar el cumplimiento y el desarrollo de las leyes. Y son estos mismos organismos quienes desplazan a las mujeres a un segundo plano en lo referente a su presencia en las diferentes instituciones. Datos que son resultado directo del compromiso de los gobernantes reflejan la distancia entre la igualdad legal y la real.

\section{Conclusiones ¿Hemos logrado nuestros objetivos?}

Como ha podido observarse, la situación de la mujer sí que ha cambiado en estos últimos años tanto desde un análisis interno como externo a ellas. Sin embargo, los objetivos marcados hace una década no se han visto cumplidos en su conjunto. Esta afirmación es confirmada a partir de diferentes fuentes documentales las cuales demuestran que España, aun habiendo mejorado aspectos relativos a la igualdad de género, no ha cumplido los objetivos que se marcó hace una década. Quizás la ambición y el positivismo fueron más fuertes que la toma de conciencia de las circunstancias sociales de aquel momento. Puede que la sociedad no estuviera preparada para una legislación tan avanzada en materia de género sin una educación específica previa. No siempre que se avanza en un determinado camino viene a significar que se han logrado los objetivos marcados. Muestra de ello es la representación de mujeres en las instituciones de representación. Ello tampoco puede dar a entender siempre que no se han invertido los esfuerzos necesarios. Los cambios sociales necesitan su tiempo para darse. Pero ello no quita para que se requiera seguir invirtiendo y trabajando en las líneas planteadas.

Los factores que afectan a la igualdad entre hombres y mujeres son diversos tanto en naturaleza como en procedencia. Por lo que requieren un análisis profundo y que trascienda de un análisis individualista. Se necesita una mirada transversal de la realidad imperante actual que mantiene algunas barreras tanto a hombres como a mujeres. No debe creerse que con la aprobación de una ley el camino ya está andado. La igualdad formal está mucho más avanzada que la social. Por ello se requiere que los esfuerzos se den a la sociedad como núcleo emisor y receptor de las desigualdades inter - géneros. Debemos 
sumar todos nuestros esfuerzos para lograr aquello que la Constitución recalca como uno de los valores jurídicos de España, que hombres y mujeres debemos ser tratados como iguales.

En definitiva, la Ley Orgánica 3/2007 inició un camino como consecuencia del esfuerzo de tantas generaciones de hombres y mujeres que nos precedieron. Estableció los pilares a partir de los cuales avanzar en materia de Igualdad de una manera transversal y panorámica. Ello hizo que abarcara una gran gama de aspectos, pero sin poder concretar en ninguno de ellos. En consecuencia, su mayor logro se convirtió en su mayor complejidad debido a que el ánimo transversal de la ley hace que sea difícil cumplir todos sus propósitos por tener un carácter ampliamente horizontal.

Tras una década de su aprobación, es el momento de evaluarla y redefinirla para dotarla de los esfuerzos y de los recursos necesarios para continuar trabajando en los objetivos que se marcó y que hoy en día siguen vigentes.

\section{Bibliografía}

BARRÈRe UnZuetA. M.A., «Igualdad y Discriminación Positiva: Un esbozo de análisis teóricoconceptual», Cuadernos electrónicos de filosofía del derecho, 9, 2003, Valencia (disponible en http://www.uv.es/cefd/9/barrereI.pdf; fecha de consulta: 2I.XII.20I6).

Bustos BotTAI, R., «Discriminación por razón de sexo y acciones positivas: reflexiones a la luz de la jurisprudencia constitucional española y aproximación a la ley para la Igualdad Efectiva», Revista de Filosofía, Derecho y Política, 6, 2007, págs.I27-I47 (disponible en http://universitas.idhbc.es/no6/06-08.pdf; fecha de consulta: 2I.XII.20I6).

Giménez Mercado, C. Valente AdARme, X., «El enfoque de los derechos humanos en las políticas públicas: ideas para un debate en ciernes». Cuadernos del Cendes, año $27 \mathrm{n}^{\circ}$ 74, Tercera Época, mayo-agosto 2010.

INSTITUTO NACIONAL DE Estadística. Anuario Estadístico 2006 (disponible en http://www.ine.es/prodyser/pubweb/anuarioo6/anuo6_o7condi.pdf; fecha de consulta I.XII.20I6).

LAMAS, M., «Diferencias de sexo, género y diferencia sexual», Cuicuilco, 7, enero-abril 2000.

Ministerio de Sanidad, Servicios Sociales e Igualdad, Determinantes de la Brecha Salarial de Género en España. Un análisis de la retribución en función de las características personales, empresariales y de la competitividad de la empresa, $20 \mathrm{or}$.

Ministerio de Trabajo y Asuntos Sociales, Anuario de Estadísticas Laborales y de Asuntos Sociales, 2006 (disponible en: http://www.empleo.gob.es/estadisticas/ANUARIO2006/welcome.htm; fecha de consulta $30 . X I .2016)$. 